\title{
Geranium Oil Algerian Type
}

National Cancer Institute

\section{Source}

National Cancer Institute. Geranium Oil Algerian Type. NCI Thesaurus. Code C107309.

The essential oil of Pelargonium graveolens. Geranium oil is used primarily for its aromatic properties. 